\title{
A campanha da catarata atrai pacientes da clínica privada?
}

\author{
Does "cataract campaign" attract private practice patients?
}

\author{
Leandro Cabral Zacharias ${ }^{1}$ \\ Rosa Maria Graziano ${ }^{2}$ \\ Bráulio Folco Telles de Oliveira ${ }^{3}$ \\ Marcelo Hatanaka ${ }^{4}$ \\ Fernando Betty Cresta $^{5}$ \\ Newton Kara José ${ }^{6}$
}

\begin{tabular}{|c|}
\hline RESUMO \\
\hline $\begin{array}{l}\text { Objetivo: Avaliar qual o tipo de assistência médica utilizada pelo paciente } \\
\text { que procura as "Campanhas da Catarata". Métodos: Foram realizadas } 299 \\
\text { entrevistas com pacientes que participaram da "Campanha da Catarata" do } \\
\text { dia } 15 \text { de abril de } 2000 \text {, no Hospital das Clínicas da Faculdade de Medicina } \\
\text { da Universidade de São Paulo. Resultados: Dos entrevistados } 58,9 \% \text { eram } \\
\text { do sexo masculinoe } 41,1 \% \text { do sexo feminino; a idade média foi de } 69,9 \text { anos. } \\
\text { A população estudada é composta principalmente por pessoas de baixa } \\
\text { escolaridade ( } 89,5 \% \text { eram analfabetas ou com escolaridade até primeiro } \\
\text { grau)e economicamente inativos em } 70,9 \% \text { (aposentados, } 50,2 \% \text {; desempre- } \\
\text { gados, } 8,3 \% \text { e donas de casa, } 12,5 \%) \text {. Os meios de assistência médica mais } \\
\text { utilizados são serviço público em } 87,0 \% \text {, convênio médico em } 8,0 \% \text { e } \\
\text { médico particular em } 4,9 \% \text {. Os principais motivos alegados pelos pacientes } \\
\text { que dispunham de convênio e não outilizaram, foram: não cobria a cirurgia } \\
\text { em } 25 \%, \text { não dava cobertura para lente intra-ocular em } 33 \% \text {. Conclusão: } \\
\text { O público alvo da "Campanha da Catarata" atendido no HC-FMUSP é } \\
\text { constituído por indivíduos de baixa escolaridade, a maioria não integrada } \\
\text { no mercado de trabalho, e que utilizam como recurso de saúde o SUS ou } \\
\text { convênios médicos que não cobrem os gastos da cirurgia de catarata. } \\
\text { Projetos catarata dentro das condições realizadas por este estudo não } \\
\text { atraem pacientes que possam pagar os custos da cirurgia de catarata. }\end{array}$ \\
\hline
\end{tabular}

Trabalho realizado pelo Departamento de Oftalmologia da Faculdade de Medicina da Universidade de São Paulo. ${ }^{1}$ Médico Residente de $1^{\circ}$ ano da Clínica Oftalmológica do Hospital das Clínicas da Faculdade de Medicina da Universidade de São Paulo - USP.

${ }^{2}$ Assitentente-Doutora da Clínica Oftalmológica do Hospital das Clínicas da Faculdade de Medicina da Universidade de São Paulo - USP.

${ }^{3}$ Médico Residente de $2^{\circ}$ ano da Clínica Oftalmológica do Hospital das Clínicas da Faculdade de Medicina da Universidade de São Paulo - USP.

${ }^{4}$ Médico Estagiário da Clínica Oftalmológica do Hospital das Clínicas da Faculdade de Medicina da Universidade de São Paulo - USP.

${ }^{5}$ Pós-Graduando da Área de Oftalmologia da Faculdade de Medicina da Universidade de São Paulo - USP.

${ }^{6}$ Professor Titular da Clínica Oftalmológica do Hospital das Clínicas da Faculdade de Medicina da Universidade de São Paulo (USP) e Universidade Estadual de Campinas (UNICAMP).

Endereço para correspondência: R. Manoel Guedes, 475 ap 114 - São Paulo (SP) CEP 04536-070.

E-mail wzacharias@uol.com.br

Recebido para publicação em 13.07.2001 Aceito para publicação em 13.03.2002

Nota editorial: Pela análise deste trabalho e por sua anuência sobre a divulgação desta nota, agradecemos ao Dr. Wallace Chamon. \begin{tabular}{l} 
INTRODUÇ̃̃o \\
\hline A perda da capacidade visual acarreta conseqüências adversas, em \\
nível individual e coletivo. A cegueira dá origem a problemas psicológicos, \\
sociais, econômicos e de qualidade de vida, pois implica em perda de auto- \\
estima, em restrições ocupacionais e consequentemente diminuição de \\
renda. Para a sociedade, representa encargo oneroso e perda de força de \\
trabalho ${ }^{(1-2)}$. \\
Estimativas da Organização Mundial de Saúde (OMS), em 1987, aponta- \\
vam a existência de 41 a 52 milhões de pessoas cegas no mundo, indicando \\
a taxa de prevalência mundial de cegueira de aproximadamente $1 \%$. A \\
catarata é considerada a principal causa de cegueira, sendo responsável \\
por, no mínimo, $50 \%$ destes casos ${ }^{(3)}$. \\
Segundo levantamentos do início da década de 90 , estimava-se haver \\
cerca de 350 mil cegos por catarata no Brasil ${ }^{(4-5)}$. Levantamentos do mesmo \\
período calculavam em 90 mil as cirurgias de catarata realizadas no Brasil \\
por ano, sendo que para atender às demandas populacionais seriam ne- \\
cessárias 450 mil cirurgias ao ano. Entre os fatores que limitam o número de \\
cirurgias, situa-se a dificuldade que a população idosa enfrenta para chegar
\end{tabular} 
ao tratamento por barreiras de ordem econômica, social e cultural $^{(3,6)}$. Considera-se, ainda, o agravante de que a população brasileira com mais de 50 anos de idade deverá aumentar de 3 a 5 vezes até o ano 2020, com aumento proporcional das necessidades de cirurgia de catarata ${ }^{(7)}$.

Deste modo, não há possibilidade de resolver o problema do acúmulo crescente dos casos não-operados a não ser com implementação de ação intensificada, integrada aos programas nacionais de prevenção de cegueira, objetivando a realização de cirurgia de catarata em grande escala, no país ${ }^{(8)}$.

As campanhas de cirurgia de catarata surgiram como um facilitador para propiciar à população carente o acesso a este tratamento. Hoje realizadas em âmbito nacional, tiveram seu início em 1987 através do "Projeto Catarata" realizado pela Disciplina de Oftalmologia da Faculdade de Ciências Médicas da Universidade Estadual de Campinas - UNICAMP ${ }^{(9)}$. Atualmente, dados do Ministério da Saúde estimam em 250 mil as cirurgias de catarata realizadas por ano no país ${ }^{(10)}$.

Na cidade de São Paulo, o Hospital das Clínicas da Faculdade de Medicina da USP (HC-FMUSP) passou a realizar as cirurgias de catarata em forma de mutirões a partir de 1996. A redução dos problemas de diminuição de acuidade visual, mais especificamente por catarata, está entre as prioridades da Disciplina de Oftalmologia, que realizou 836 cirurgias de catarata em 1998 e 2361 cirurgias em1999 (dados do Serviço de Arquivo Médico do HC - FMUSP).

O número de cirurgias de catarata realizadas no Brasil aumentou de 90 mil no começo da década de 90 para aproximadamente 250 mil em 2000; esse aumento de $278 \%$ é extraordinário e se deve a um esforço conjugado dos oftalmologistas e autoridades de saúde ${ }^{(10)}$. Os mutirões de catarata foram instrumento usado para divulgar os benefícios da cirurgia, a existência da demanda reprimida e de facilitação do acesso da população menos favorecida economicamente à cirurgia. Passados 14 anos desde a sua introdução, deve-se questionar da validade de sua continuação: existiria ainda demanda reprimida, o projeto estaria priorizando a população carente, a conscientização e educação da população já permitiriam um livre acesso à cirurgia?

Realizou-se o presente estudo para avaliar os recursos médicos disponíveis aos pacientes que procuram "Campanhas de Catarata" para realizar sua cirurgia.

\section{MÉTODOS}

Foi realizado um mutirão de catarata no dia 15 de abril de 2000, no Prédio dos Ambulatórios do HC-FMUSP. A divulgação foi realizada por meio de panfletos, faixas, anúncios em jornais, rádio e televisão. A triagem inicial consistiu em avaliar a acuidade visual (AV) com a correção óptica que o paciente usava, biomicroscopia do segmento anterior do olho e tonometria de aplanação. Indivíduos com AV melhor ou igual a 0,4 foram dispensados dos exames seguintes. Os indivíduos com AV pior que 0,4 foram submetidos a exame de refração, motili- dade ocular, fundo de olho e biomicroscopia do segmento anterior. Quando não se visualizava o fundo de olho, foi realizada ecografia para afastar comprometimento vítreo-retiniano. Os casos com indicação cirúrgica tiveram o grau da lente intra-ocular calculado através de biometria ultra-sônica.

Após receberem os pedidos de exames pré-operatórios, os pacientes selecionados para cirurgia de catarata foram registrados no Hospital das Clínicas da Faculdade de Medicina da Universidade de São Paulo (HC - FMUSP), receberam os pedidos de exame pré-operatório e agendamento do dia da cirurgia. Receberam ainda orientações com relação aos cuidados pré e pós-operatórios. Os questionários analisados no presente trabalho foram aplicados a seguir.

Foram atendidos neste dia 2082 pacientes, sendo que 801 cirurgias de catarata foram agendadas (dados do Serviço de Arquivo Médico do HC - FMUSP). Os autores aplicaram 299 questionários em pacientes selecionados por conveniência (aplicação de questionários dentro das limitações de tempo em que foi realizado o estudo).

Questões de múltipla escolha (Tabela 1) foram aplicadas verbalmente pelos examinadores e as respostas avaliadas

\section{Tabela 1. Questionário aplicado aos pacientes}

Nome:

Idade:

Sexo:

Escolaridade:

O Sr(a) trabalha? Que tipo de trabalho? Aposentado?

1 - Quando o $\operatorname{Sr}(\mathrm{a})$ necessita de atendimento médico, procura: a - Serviço médico particular

b - Serviço médico de convênio

c - Serviço público Qual serviço público? E'funcionário público? d - Sindicato patronal

2 - Caso o $\mathrm{Sr}(\mathrm{a})$ utilize convênio/sindicato

- Qual é o seu convênio / sindicato?

- Há quanto tempo o Sr(a) tem convênio / sindicato?

- O seu convênio é:

a) Empresarial

b) Particular (é você quem paga o convênio)

c) Você paga percentual do tratamento

3 - O Sr(a) já sabia que tinha de ser operado de catarata? Em caso afirmativo, há quanto tempo?

4 - Quem fez o diagnóstico de sua catarata?

a - Médico particular

b - Médico de convênio

c - Médico de serviço público

d - Outra campanha de Catarata

e - O diagnóstico foi feito nesta consulta

5 - No caso de a cirurgia ter sido indicada anteriormente, não a realizou por qual dos motivos:

a - Período de carência do convênio

b - Convênio não cobria a cirurgia

c - Convênio não cobria a lente intra-ocular

d - Falta de condição clínica para a cirurgia

e - Marcação cirúrgica muito demorada

f - Outras 
quanto aos dados sociodemográficos e o tipo de assistência médica que os entrevistados dispunham.

A tabulação dos dados, análise dos resultados e confecção de gráficos foi realizada através dos programas EpiInfo 6 vs 6.04b e MS Excel 97.

\section{RESULTADOS}

Dos entrevistados, $176(58,9 \%)$ eram do sexo masculino e $123(41,1 \%)$ do sexo feminino. A idade média foi de 69,9 anos, sendo o desvio-padrão de 9,6 e a mediana de 67 anos.

Quanto à escolaridade, a maioria $(60,7 \%)$ cursou somente o $1^{\circ}$ grau (até a oitava série), e $28,8 \%$ dos entrevistados eram analfabetos. Somente dois $(0,8 \%)$ tinham curso superior (formação universitária) (Gráfico 1).

Dos 289 pacientes que responderam à pergunta sobre atividade ocupacional atual, pouco mais da metade eram aposentados $(50,2 \%), 8,3 \%$ estavam desempregados, $12,5 \%$ eram "donas de casa" e 5,2\%, empregadas domésticas.

Inquiridos sobre o tipo de assistência médica que utilizavam mais frequientemente, 260 pacientes $(87,0 \%)$ responderam que procuram o serviço médico público, 24 pacientes $(8,0 \%)$ convênios médicos, 14 pacientes $(4,7 \%)$ a rede particular e 1 paciente $(0,3 \%)$ um sindicato patronal. (Gráfico 2$)$

Do total de entrevistados, $24(8,1 \%)$ utilizavam convênios médicos. Destes, dois terços (16) tinham planos particulares e um terço (8), planos empresariais.

O gráfico 3 ilustra as razões pelas quais os 24 pacientes que dispunham de convênio não o utilizaram para realização de sua cirurgia. Destes pacientes, $33 \%$ alegaram que o convênio não cobria a lente intra-ocular, em $25 \%$ o convênio não cobria a cirurgia, $17 \%$ não sabiam que tinham catarata, $8 \%$ afirmaram que a marcação da cirurgia era demorada e $4 \%$ alegaram estar dentro do período de carência.

Dos 299 entrevistados, 84 (28,1\%) não sabiam que apresentavam catarata. Dos 215 pacientes restantes, $131(60,9 \%)$ tiveram o diagnóstico feito na rede pública, 49 (22,8\%) por médico particular, $27(12,6 \%)$ em médico de convênio e oito $(3,7 \%)$ realizados em serviços públicos em "Campanhas da

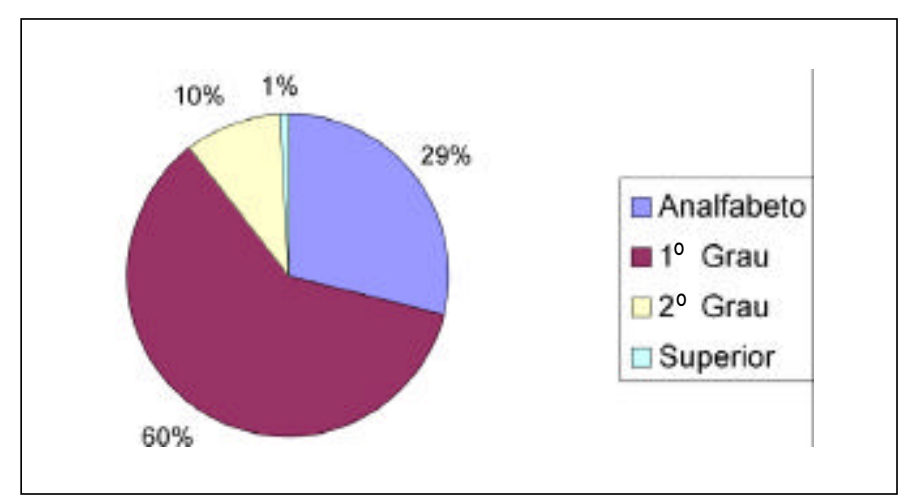

Gráfico 1 - Escolaridade dos Entrevistados

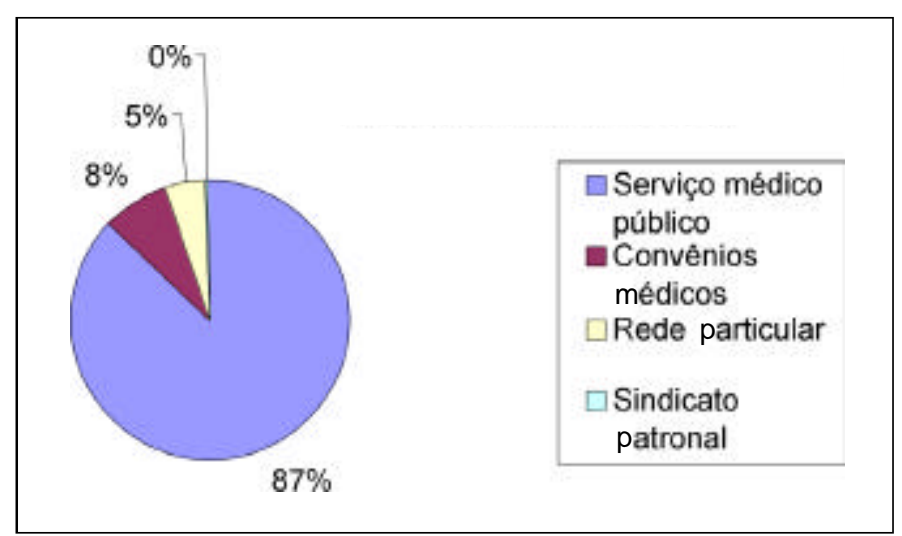

Gráfico 2 - Assistência médica mais comumente utilizada pelos entrevistados

Catarata" anteriores. Assim, 74,6\% dos diagnósticos de catarata foram feitos pela rede pública, $9,0 \%$ através de convênios médicos e 16,4\% através de atendimento particular.

\section{DISCUSSÃO}

Os dois sexos foram entrevistados de forma semelhante e a faixa etária composta principalmente por pessoas acima de 60 anos de idade (média 69,9 anos). Outros trabalhos mostram em nosso meio que a catarata avançada é mais comum após os 60 anos e igualmente distribuída entre os sexos ${ }^{(11-12)}$.

A população do Projeto Catarata é composta na sua maioria por pessoas de baixa escolaridade $(89,50 \%$ eram pessoas analfabetas ou com escolaridade até o primeiro grau) e economicamente inativas em 70,93\% (aposentados, 50,17\%, desempregados, $8,30 \%$ e donas de casa, $12,46 \%$ ). Em projeto anterior foi observado que a população economicamente ativa selecionada para cirurgia em Projetos Catarata constituía apenas $23,07 \%$ da amostra ${ }^{(12)}$, dado que sugere inatividade precoce devido à diminuição de acuidade visual.

Quando inquiridos sobre qual a forma de assistência médica que mais freqüentemente utilizam quando necessitam, a maioria utiliza o sistema público de saúde $(87,0 \%)$. Convênios

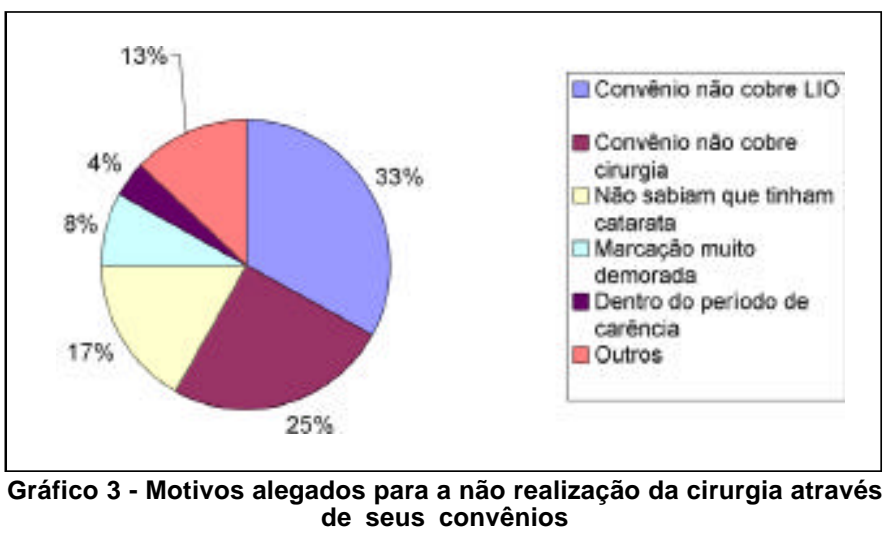


médicos são utilizados por 8,0\% e o atendimento particular por $4,7 \%$ dos entrevistados. Estes dados coincidem com a estimativa feita de que $3 \%$ da população brasileira tem acesso à medicina particular, $17 \%$ a convênios médicos e os restantes $80 \%$ apenas à assistência médica pública ${ }^{(5)}$.

Setenta e dois por cento dos entrevistados já possuíam diagnóstico prévio de catarata, e não conseguiram chegar à cirurgia. Essa ocorrência têm sido constante em Projetos "Zona Livre de Catarata"(12). Quanto ao diagnóstico de catarata, $74,6 \%$ foi realizado na rede pública, $9 \%$ pelo convênio e 16,4\% em médico particular. A porcentagem de diagnósticos feito em médico particular poderia sugerir que alguns pacientes utilizam a rede pública como forma principal de atendimento médico e que seu recurso financeiro seria suficiente para uma consulta ocasional mas não para a realização do procedimento cirúrgico ou mesmo para custear mensalmente um plano de saúde ${ }^{(12)}$.

Nenhum paciente com plano de seguro com validade para cobrir todos os gastos da cirurgia procurou o atendimento gratuito do projeto. Quanto aos motivos pelos quais os pacientes que dispunham de plano de saúde $(8,0 \%$ dos entrevistados) não o utilizaram para a cirurgia, $4 \%$ deste grupo estava ainda em período de carência; o convênio não cobria as cirurgias ou a demora na marcação cirúrgica impediu que 33\% dos pacientes tivessem sua cirurgia realizada; em $33 \%$ dos casos o convênio não cobria lente intra-ocular e o paciente não tinha condições de adquirí-la.

Estudo envolvendo o "Projeto Catarata" realizado na cidade de São Manuel mostram que 93,18\% não teriam condições de realizar a cirurgia de outra forma que não através do projeto. Os mesmos autores referem que $78,84 \%$ dos entrevistados que sabiam ter catarata não foram operados por falta de recursos financeiros ${ }^{(12)}$. Analisando a população de cinco municípios do estado de São Paulo ("Projeto Zona Livre de Catarata") foi demonstrado que em $69,3 \%$ as dificuldades financeiras foram responsáveis pela não realização da cirurgia anteriormente, e concluem que as barreiras para a realização da cirurgia de catarata continuam presentes, de modo que Projetos "Zona Livre de Catarata" continuam sendo necessários em nosso meio ${ }^{(11)}$.

Vários fatores podem explicar porque as "Campanhas da Catarata" em Hospitais-Escola atraem um grande número de pacientes.

1. A divulgação entre a população de que a cegueira por catarata é reversível, e que a cirurgia apresenta bons resultados;

2. A imagem de respeitabilidade e competência médica que Hospitais Universitários que mantém projetos assistenciais como as "Campanhas de Catarata" mantém junto à população;

3. O contexto sócio-econômico atual do país. Estima-se que em 1993, o Sistema Único de Saúde foi responsável por $80 \%$ das cirurgias de catarata realizadas no país ${ }^{(5)}$;

4. A quebra das barreiras ao acesso à cirurgia através das "Campanhas de Catarata", fazendo com que o paciente consiga atendimento e resolução de sua condição cirúrgica, atraindo desta forma os pacientes mais carentes.

A avaliação do Projeto Catarata em dois momentos diferentes em duas cidades diferentes (São Manuel, 1994 e São Paulo, 2000) apresentou resultados concordantes quanto a: faixa etária de acometimento acima dos 60 anos, inatividade econômica dos pacientes selecionados no projeto, e dificuldade de realização de cirurgia de catarata mesmo entre os pacientes com diagnóstico prévio realizado por médico particular ou convênio médico. Logo, os dados obtidos neste estudo são concordantes com os obtidos em São Manuel e sugerem que Projetos Catarata realizados dentro das condições de São Manuel e São Paulo não atraem pacientes que possam pagar a cirurgia.

\section{CONCLUSÕES}

1. O público alvo da "Campanha de Catarata" é constituído por indivíduos de baixa escolaridade economicamente carentes e não integrados ao mercado de trabalho em sua maioria.

2. $86 \%$ dos indivíduos que procuram o projeto catarata utilizam o sistema público de saúde para seu tratamento.

3. Nenhum paciente com outro tipo de atendimento médico que cobrisse os gastos da cirurgia procurou o mutirão de catarata.

4. As "Campanhas de Catarata" já se firmaram como ações efetivas e demonstrativas das condições de atendimento da população idosa e carente até que o Sistema de Saúde tenha condições de permitir de rotina o acesso a tratamento de todos os necessitados.

5. Projetos catarata dentro das condições realizadas por este estudo não atraem pacientes que possam pagar os custos da cirurgia de catarata.

\section{ABSTRACT}

Purpose: To evaluate which kind of medical care cataract patients have. Methods: The authors of the survey conducted 299 interviews with patients who took part in the "Cataract Campaign" on April 15, 2000. Results: Among the interviewees, $58.9 \%$ were males, and $41.1 \%$ were females, with an average age of 69.9 years. Our population consisted largely of low educational level people $(89.5 \%$ between illiteracy and elementary school level), and $70.9 \%$ are economically inactive (retired, $50.2 \%$; unemployed, $8.3 \%$; housewives $12.5 \%$ ). The kind of medical care most frequently used was public health service (87.0\%); followed by health insurance companies (8.0\%) and private care $(4.9 \%)$. The main reasons reported by patients who had health insurance but had not used it for the surgery were: the companies would not pay for the surgery (25.0\%), would not provide the intraocular lens (33.0\%). Conclusion: We conclude that the target public in the "Cataract Campaign" comprises low educational level individuals, most of them economically inac- 
tive. Even those who have health insurance seek for help in the campaign due to the lack of economical conditions. In the conditions of this study, the "Cataract Campaign" does not attract people who can pay for the surgery.

Keywords: Cataract/pathology; Visual acuity; Medical assistance/utilization; Socioeconomic factors; Community health services; Human; Aged

\section{REFERÊNCIAS}

1. Temporini, ER, Kara-José N. Níveis de prevenção de problemas oftalmológicos: propostas de investigação. Arq Bras Oftalmol 1995;58:189-92.

2. Thylefors, B. Much blindness is avoidable. World Health Forum 1991;12:78-86.

3. Schwalbe, L. Cataract blindness in developing nations. Int Ophthalmol Clin 1990;30:16-8.

4. Kara José N, Delgado AMN, Arieta CEL. Catarata. [Tema oficial ]. In: XI Congresso Brasileiro de Prevenção da Cegueira.; 1994; Brasília. Anais. 1994. p.17 e 18 .
5. Kara José,N, Alves MR. Catarata: Um problema de saúde pública. In: KaraJosé N. (Organizador) Prevenção da cegueira por catarata. Campinas: UNICAMP; 1996. p.11-8.

6. Alves MR, Kara-José N, Silva ALB, Prado Jr J. Temporini ER. Características e percepções de pacientes portadores de catarata senil atendidos em projeto comunitário de reabilitação visual. Rev Hosp Clin Fac Med Univ São Paulo 1997;52:6-19.

7. Kara-José N, Delgado NA, Arieta CEL. Catarata In: Rodrigues MLV. Oftalmologia Clínica. Rio de Janeiro: Cultura Médica; 1992. p.288-302.

8. Ungaro ABS, Vilella FF, Klein RCA, Kara-José N, Alves MR, Crest, FB. Campanha Nacional de Prevenção da Cegueira e Campanha Nacional de Reabilitação do Idoso. Experiência no Hospital das Clínicas da Faculdade de Medicina da Universidade de São Paulo. Rev Med São Paulo 1997;76:97-100.

9. Temporini, ER, Kara-Jose N; Kara-José Jr N. Catarata senil: características e percepções de pacientes atendidos em projeto comunitário de reabilitação visual. Arq Bras Oftalmol 1997;60:79-83.

10. País realiza 120 mil cirurgias extras de catarata. Jornal Jota Zero 1999; nov/ dez. p.23.

11. Kara José N, Temporini ER. Cirurgia de catarata: o porque dos excluídos Rev Panam Salud Pública 1999;6:242-8.

12. Kara José Jr N, Schellini AS, Silva MRBM, Bruni LF, Almeida ACG Projeto Catarata - Qual a sua importância para a comunidade? Arq Bras Oftalmol 1996;59:490-3.

\title{
Congresso da ALACCSA
}

\author{
06 e 07 de Dezembro de 2002 \\ PUNTA DEL ESTE - URUGUAI
}

INFORMAÇÕES: Secretariat:GRCongresos\& Reuniones

Tel.: (598-2) 9160900

Fax.: $(598-2) 9168902$

E-mail: risu@adinet.com.uy/alaccsa@rohrsa.com

Home page: www.alaccsa.com/www.congresos-rohr.com 